\title{
The observing response is not due to biased expectancies'
}

THOMAS S. WALLSTEN and JACK CATLIN, Department of Psychology, University of Pennsylvania, Philadelphia, Pa.

A modified probability learning experiment with humans tested the hypothesis that the observing response (OR) occurs because $S$ mistakenly expects the preferable event more often following the $O R$ than the $\overline{O R}$. The $O R$ effect was clearly present but evidence of biased expectancies was not. These results lend weight to motivational theories, which posit greater utility of events following the $O R$ than the $\overline{O R}$.

Wykoff (1952) was the first to demonstrate that Ss will perform an observing response (OR) to gain information, i.e., a response that produces stimuli predictive of the outcomes of an instrumental response (IR) but does not control these outcomes. This has since been shown with a variety of species in various situations (e.g., Prokasy, 1956; Kelleher, 1958; Bower, McLean, \& Meacham, 1966).

Explanations of the phenomenon have been in terms of motivation; the $O R$ is made because subsequent events have greater utility than when the $\overline{\mathrm{OR}}$ is made. For example, stimuli predictive of valued outcomes may have intrinsically greater utility than unpredictive stimuli. Alternatively, events contingent on the IR may have greater utility when the IR follows the $O R$ than when it follows the $\overline{O R}$. One mechanism for the latter possibility has been proposed by Perkins (1955). He suggested that the predictive stimuli following the OR allow $\mathbf{S}$ to make preparatory responses which increase the utility of the outcomes. ${ }^{2}$

Motivational theories can be weighed against an expectancy theory. Such an expectancy theory postulates that the $O R$ is made because S's expectations are biased by the differential feedback following the $O R$ and $\overline{O R}$, so that he expects the preferable event more of ten following the $\mathrm{OR}$ than the $\overline{\mathrm{OR}}$. Thus the $O R$ is made in an attempt to maximize subjective likelihood of obtaining the preferable event. It has been shown in related contexts that expectancies can be biased by outcome structures (see Irwin \& Snodgrass, 1966). This paper presents an experiment with humans to test the expectancy theory of the OR.

Imagine an experiment in which $S$ sequentially predicts whether a preferable or a neutral event will occur. The preferable event has a positive independent outcome $(+\mathrm{IO})$ associated with it, and the neutral event has none, i.e., when the former occurs, $S$ receives a prize regardless of his prediction. In addition, after each prediction $S$ receives a dependent outcome (DO) the value of which depends on whether he predicted correctly. The event to occur does so with a fixed delay after the prediction.

The $\mathbf{S}$ makes his prediction by pushing one button of either of two pairs. When he uses the informative pair of buttons (I-pair), he is immediately signaled whether he is correct. When he uses the noninformative pair of buttons ( $N$-pair), he is not signaled during the delay period. If $S$ has a tendency to make the $\mathrm{OR}$ rather than the $\widehat{\mathrm{OR}}$, he will demonstrate a preference for making predictions with the I-pair. If biased expectancies are the cause of the OR effect, $S$ will predict the preferable event more often with the I-pair than with the N-pair. This experiment was done.

\section{METHOD}

The apparatus, diagrammed in Fig. 1, was $30 \times 30$ in. Red trial-start lights are indicated by the letter $A$, buttons with

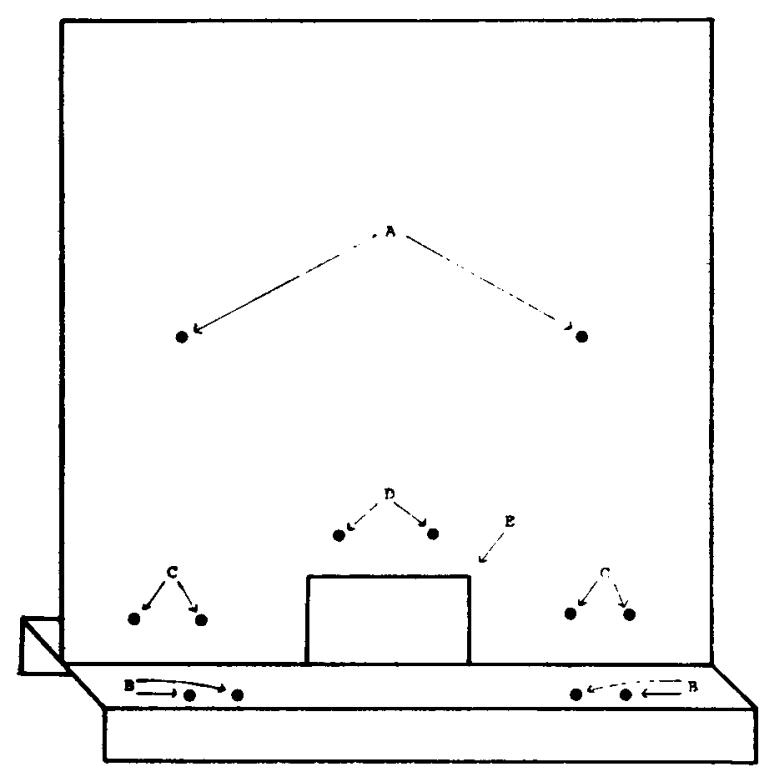

Fig. 1. Diagram of apparatus. See text for details.

which the $S$ makes his predictions by $B$, white feedback lights by $C$, white event lights by $D$, and a cliute for dispensing tokens by $\mathrm{E}$.

The token dispenser released through the chute either or both of two distinct types of tokens. One type, steel washers $3 / 4$ in. in diameter and $3 / 32$ in. thick, represented the +10 of $11 / 2 \mathrm{c}$. The other type, brass washers $1 / 2 \mathrm{in}$. in diameter and $1 / 16$ in. thick, represented the DO of ic for correct predictions. (The DO for incorrect predictions was Oc.) $\mathrm{S}$ accumulated his tokens on spindles.

The event which $S$ was to predict on each trial was the flashing of one of the event lights. Using one of the pairs of buttons, $S$ predicted the left or right event by pushing the button in the corresponding position of that pair. The events were equally likely and random, and one or the other occurred for 2 sec on each trial. The appropriate tokens were dispensed simultaneously with the events. Each of the events was made preferable for half the Ss.

The delay between S's prediction and the event was $10 \mathrm{sec}$. One of the feedback lights above the pair of buttons used was turned on for the duration of the interval. When $S$ used the pair of buttons designated as the I-pair, the position of the feedback light which illuminated was perfectly correlated with the subsequent event. When he used the N-pair, this correlation was zero. Each pair of buttons was the I-pair for half the Ss.

The session included two types of trials, forced and choice. A forced trial was denoted by the illumination of one of the trial-start lights; only the pair of buttons below that light was operative. A choice trial was denoted by the illumination of both trial-start lights; $S$ could choose the pair of buttons with which to make his prediction. On both forced and choice trials $S$ was free to make his prediction at any time after the trial-start light(s) turned on. When he did so they turned off. Immediately following the occurrence of the event, trial-start lights initiated the next trial. 
Table 1

Per Cent Predictions Made with I-Pair Buttons During Choice Trials, and Per Cent Predictions of the Preferable Event During Forced Trials with I-Pait and N-Pair Buttons

\begin{tabular}{|c|c|c|c|c|c|c|c|c|c|}
\hline Subjects & 1 & 2 & 3 & 4 & 5 & 6 & 7 & 8 & Mean \\
\hline I-pair choices & 94 & 96 & 100 & 56 & 74 & 72 & 66 & 84 & 71 \\
\hline $\begin{array}{c}\text { Preferable- } \\
\text { 1-pair }\end{array}$ & 61 & 35 & 48 & 48 & 43 & 51 & 52 & 57 & 50 \\
\hline $\begin{array}{c}\text { Preferable- } \\
\text { N-pair }\end{array}$ & 77 & 27 & 41 & 51 & 52 & 25 & 53 & 58 & 48 \\
\hline
\end{tabular}

The experimental session, which was preprogrammed and automatically controlled, consisted of a series of blocks of 50 forced trials each, followed by 50 choice trials. $S$ was forced to use one pair of buttons throughout a given block of forced trials. The side to which $\mathrm{S}$ was forced alternated from block to block. Four random sequences consisting of 10 blocks of 50 forced trials each were constructed. Using each sequence from either end resulted in eight different sequences. Half of these sequences began with the I-pair and half with the $\mathrm{N}$-pair of buttons. In addition a block of 50 choice trials, to follow the forced trials, was constructed for each $S$.

The eight Ss, recruited from an introductory psychology class, were fully informed about the task. They were told about the DOs, the $+\mathrm{IO}$, and which event was preferable. The difference between the I-pair and $\mathrm{N}$-pair buttons was explained, but each $S$ had to discover which pair was the l-pair. Before the choice block, Ss were reminded that they could choose the pair of buttons with which to make a prediction on each trial.

An $\mathrm{S}$ served in each of the cells defined by the intersection of two positions for the l-pair buttons by two positions for the preferable event by two types of beginning forced blocks. The initial plan was to run each $S$ through 10 blocks of forced trials, alternating between I-pair and $\mathrm{N}$-pair buttons, followed by his block of choice trials, with a rest period after Blocks 5 and 10. After Ss 1,2, and 3, it became apparent that sufficiently stable behavior was achieved prior to Block 6 . The remaining Ss were run through the first six blocks of forced tiials and then linrough the block of choice trials, with a rest after Blocks 3 and 6 . Ss were paid strictly according to the tokens they had won.

\section{RESULTS AND DISCUSSION}

The results are shown in Table 1 . The top row of the table gives the per cent of the trials in the 50-trial choice block on which each $S$ made his predictions with the I-pair buttons. All Ss chose to use that pair for over half their predictions, indicating the presence of the OR phenomenon.

The bottom two rows of the table give the per cent of the trials on which each $S$ predicted the preferable event for the 150 trials during which he was forced to use the I-pair buttons and for the 150 trials during which he was forced to use the $\mathrm{N}$-pair buttons. In the case of Ss 1,2 , and 3, only the first 150 trials with either pair of buttons were used for the analysis. However, the comparable percentages based on all 250 trials were virtually the same. They were 60,41 , and 49 for Ss 1,2 , and 3 , respectively, with the 1-pair buttons and 76, 30, and 44 for Ss 1, 2, and 3, respectively, with the N-pair buttons. It is obvious that the preferable event was not predicted significantly more often with the I-pair buttons than with the $\mathrm{N}$-pair. Five of the eight Ss predicted it less often with the 1-pair, and the overall mean per cent predictions are virtually identical under the two conditions.

The absence of evidence for the postulated biased expectancies in the group data leaves open the possibility of a correlation between the degree of the OR effect and the degree to which the preferable event was predicted more often with the I-pair than with the N-pair buttons. Spearman's rho was calculated between these two variables to ascertain whether such a correlation existed. The former variable was measured by the number for each $S$ in the top row of Table 1 , and the latter variable was measured by the ratio of the two numbers for each $S$ in the bottom rows of the table. The rank order correlation was .21 , which did not approach significance. Without Ss 1, 2, and 3, it was .20.

All Ss demonstrated the OR phenomenon. The hypothesis that this was caused by biased expectancies was clearly not supported. The biased expectancy hypothesis was proposed as a strong alternative to the entire class of motivational theories. To the extent that the OR phenomenon was demonstrated firmly in this study while biased expectancies were not, support is indirectly given to the latter class of theories.

It is interesting that Ss 1,2 , and 3, who were forced to work at the task for 200 trials longer than the other $\mathrm{Ss}$, demonstrated the strongest tendencies to make the OR. This suggests that in the present experiment the degree of $O R$ effect depended on the length of time, or number of trials, in the task. All Ss reported the session to be quite boring, which was the main reason for reducing the number of trials. Perhaps boredom increased the tendency to choose the same pair of buttons for all predictions, thus augmenting the apparent size of the OR effect.

\section{REFERENCES}

BOWER, G., McLEAN, J., \& MEACHAM, J. Value of knowing when reinforcement is due. Journal of Comparative \& Physiological Psychology, 1966, 62, 184-192.

IRWIN, F. W., \& SNODGRASS, JOAN GAY. Effects of independent and dependent outcome values upon bets. Journal of Experimental Psychology, 1966, 71, 282-285.

KELLEHER, R. T. Stimulus-producing responses and attention in the chimpanzee. Journal of the Experimental Analysis of Behavior, 1958, 1,87-102.

PERKINS, C. C., Ir. The stimulus conditions which follow learned responses. Psychological Review, 1955, 62, 341-348.

PROKASY, W. F., Jr. The acquisition of observing responses in the absence of differential external reinforcement. Journal of Comparative \& Physiological Psychology, 1956, 49, 131-134.

WYKOFF, L. B. The role of observing responses in discrimination learning. Psychological Review, 1952, 59, 431-442.

WYKOFF, L. B. Toward a quantitative theory of secondary reinforcement. Psychological Review, 1959, 66, 68-78. NOTES

1. This study was supported by PHS Grant No. MH-06580 to Francis W. Irwin. The authors thank F. W. Irwin for his comments and advice throughout the course of the work, and Jon Ritt for collecting the data. The first author is a USPHS Predoctoral Fellow and the second is a Danforth Fellow.

2. Wykoff's (1959) model and the general analysis by Bower et al. (1966) explain the OR effect in terms of increased secondary reinforcement, or utility, of the stimuli immediately following the OR. However, as Bower et al point out, this explanation is neutral with regard to the mechanism underlying this increase in utility of the predictive stimuli over the unpredictive stimuli. 